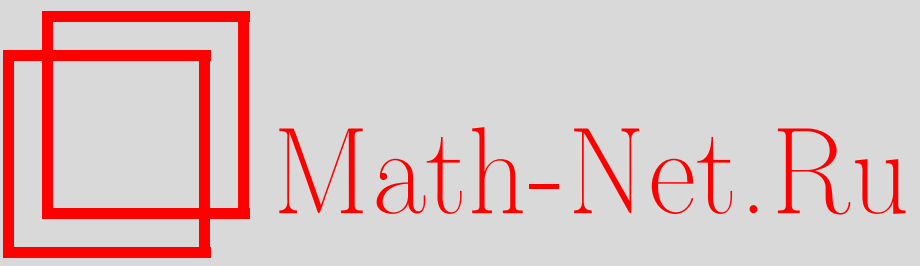

Д. А. Заусаев, Л. А. Соловьёв, Численное интегрирование уравнений движения небесных тел с учётом регуляризации и использованием оскулирующих элементов больших планет, Вестн. Сам. гос. техн. ун-та. Сер. Физ.-мат. науки, 2010, выпуск 5(), 305-308

DOI: https://doi.org/10.14498/vsgtu807

Использование Общероссийского математического портала Math-Net.Ru подразумевает, что вы прочитали и согласны с пользовательским соглашением http: //www . mathnet.ru/rus/agreement

Параметры загрузки:

IP : 54.224 .135 .184

26 апреля 2023 г., 13:59:33 
Небесная механика и астрономия

УДК 521.182, 521.642

\title{
ЧИСЛЕННОЕ ИНТЕГРИРОВАНИЕ УРАВНЕНИЙ ДВИЖЕНИЯ НЕБЕСНЫХ ТЕЛ С УЧЁТОМ РЕГУЛЯРИЗАЦИИ И ИСПОЛЬЗОВАНИЕМ ОСКУЛИРУЮЩИХ ЭЛЕМЕНТОВ БОЛЬШИХ ПЛАНЕТ
}

\author{
Д. А. Заусаев, Л. А. Соловъев \\ Самарский государственный технический университет, \\ 443100, Самара, ул. Молодогвардейская, 244. \\ E-mail: zadmitriy@gmail.com
}

Получены регулярные дифберенциальные уравнения для задачи возмущенного движения. Разработаны вычислительный алгоритм и программа численного интегрирования уравнений движения небесных тел методом Эверхарта с учётом регуляризации и использованием оскулирующих элементов больших планет.

Ключевые слова: интерполячия, регуляризация, численное интегрирование, дифберенциальные уравнения двиюения, метод оскулирующих элементов.

Численное интегрирование уравнений движения малых тел Солнечной системы проводится путем совместного решения уравнений движения больших планет и исследуемого объекта. При решении задач, связанных с проблемой астероидной опасности, требуется проводить регулярные исследования движения свыше 7000 объектов на интервале времени порядка нескольких столетий. Создание высокоэффективных алгоритмов и программ численного интегрирования уравнений движения небесных тел является необходимым условием для оперативного получения результатов проведенных исследований. В настоящее время требования к точности координат больших планет существенно возросли, так как они должны быть согласованы с высокоточными астрономическими наблюдениями.

Целью данной работы является увеличение точности координат планет с помощью банка данных оскулирующих элементов.

Разработаны вычислительный алгоритм и программа, реализующая численное интегрирование уравнения движения небесного тела с использованием оскулирующих элементов больших планет [1].

Основная идея реализации данного алгоритма заключается в следующем. На основе численной теории движения планет DE405 [2] создан банк данных оскулирующих элементов орбит больших планет и Луны с шагом 1 день на интервале времени с 1600 по 2200 гг. Координаты и скорости планет и Луны на любой момент времени внутри вышеуказанного промежутка вычисляются с помощью невозмущенных кеплеровских орбит.

Создание банка данных координат больших планет способствует понижению порядка системы дифференциальных уравнений с 72 до 6, что существенно сокращает расчётное время по сравнению с совместным интегрированием.

Дифференциальные уравнения движения небесного тела в гелиоцентрической системе координат с учётом релятивистских эффектов от Солнца имеют следующий вид [3]:

Дмитрий Анатольевич Заусаев, аспирант, каф. прикладной математики и информатики. Леонид Александрович Соловъев, аспирант, каф. прикладной математики и информатики. 


$$
\begin{aligned}
\frac{d^{2} X}{d t^{2}}= & -k^{2}(1+m) \frac{X}{r^{3}}+\sum_{i} k^{2} m_{i}\left(\frac{X_{i}-X}{\Delta_{i}^{3}}-\frac{X_{i}}{r_{i}^{3}}\right)+ \\
& +\frac{k^{2}}{c^{2}}\left[(4-2 \alpha) \frac{k^{2}}{r^{4}} X-(1+\alpha) \frac{\dot{r}^{2}}{r^{3}} X+3 \alpha \frac{(X \dot{X})^{2}}{r^{5}} X+(4-2 \alpha) \frac{(X \dot{X})}{r^{3}} \dot{X}\right]
\end{aligned}
$$

где $X$ - матрица-столбец с элементами $x, y, z ; X_{i}$ - матрица-столбец с элементами $x_{i}, y_{i}, z_{i} ; m, x, y, z$ - масса и гелиоцентрические координаты возмущаемого тела; $m_{i}, x_{i}, y_{i}, z_{i}$ - массы и гелиоцентрические координаты больших планет; $r, \Delta_{i}, r_{i}-$ расстояния, вычисляемые по формулам $r^{2}=x^{2}+y^{2}+z^{2}, \Delta^{2}=\left(x_{i}-x\right)^{2}+\left(y_{i}-\right.$ $-y)^{2}+\left(z_{i}-z\right)^{2}, r_{i}^{2}=x_{i}^{2}+y_{i}^{2}+z_{i}^{2} ; \dot{X}$ - матрица-столбец с элементами $\dot{x}, \dot{y}, \dot{z} ;$ $k$ - постоянная Гаусса, $c$ - скорость света, $\alpha$ - параметр, характеризующий выбор системы координат. Случай $\alpha=1$ соответствует стандартным координатам, случай $\alpha=0$ - гармоническим координатам.

Дифференциальное уравнение (1) можно представить в более компактном виде:

$$
\ddot{x}+\frac{K^{2}}{r^{3}} x=-\frac{\partial V}{\partial x}+P
$$

где $x$ - вектор положения возмущаемого тела, $V$ - возмущающий потенциал, $P$ дополнительная сила, $K^{2}=k^{2}(M+m), r=|x|, M$ - масса центрального тела, $m-$ масса возмущаемого тела. Уравнения (2) являются сингулярными в начале координат. При прохождении тела вблизи начала координат возникают большие гравитационные силы и происходит резкое изменение орбиты. При численном интегрировании для преодоления этой трудности приходится значительно уменьшать длину шага интегрирования, что приводит к увеличению ошибок округления. Это существенно сказывается на точности результатов проведенных расчётов.

Процедура преобразования сингулярных уравнений в регулярные уравнения называется регуляризацией. Регуляризация уравнений (2) проводится в два этапа. На первом этапе путём введения новой независимой переменной $s$, называемой фиктивным временем, получают регулярные функции, описывающие движение.

Второй этап регуляризации заключается в устранении сингулярности в самих дифференциальных уравнениях. Это достигается путем преобразования вектора физического пространства в четырехмерное параметрическое пространство с помощью матрицы преобразования Кустаанхеймо [4].

Применяя процедуру регуляризации к уравнению (1), получим следующую систему дифференциальных уравнений [4]:

$$
\left\{\begin{array}{l}
u^{\prime \prime}+\frac{h_{k}}{2} u=\frac{|u|^{2}}{2}\left(-\frac{1}{2} \frac{\partial V}{\partial u}+L^{\top} P\right), \\
h_{k}^{\prime}=\left(\frac{\partial V}{\partial u}, u^{\prime}\right)-2\left(u^{\prime}, L^{\top} P\right), \quad t^{\prime}=(u, u),
\end{array}\right.
$$

где $u$ - четырёхмерный вектор с параметрами $u_{1}, u_{2}, u_{3}, u_{4} ; h_{k}=\left(K^{2}-2\left|u^{\prime}\right|^{2}\right) /|u|^{2}-$ уравнение энергии; $L^{\top}$ - транспонированная матрица преобразования Кустаанхеймо [4]; штрих означает дифференцирование по $s$.

Регуляризация уравнений (2) проводилась в два этапа. Сначала были получены дифференциальные уравнения возмущаемого тела с учётом гравитационных и релятивистских эффектов, обусловленных Солнцем, относительно новой независимой переменной $s[4]$. Координаты планет и Луны при численном интегрировании определялись с помощью банка данных DE405. Эти уравнения являются сингулярными. Затем с помощью преобразования координат были получены регулярные уравнения, подобные уравнениям (3).

Дифференциальные уравнения (3) регулярны, по сравнению с уравнениями (2) они обладают лучшей устойчивостью. Отмечая наиболее существенное преимуще- 
ство уравнений (3) по сравнению с классическими уравнениями, следует также отметить, что их можно использовать для исследования движения небесных тел, имеющих любые эксцентриситеты и сближения с большими планетами.

Однако при совместном численном интегрировании уравнений движения больших планет и возмущаемого тела возникают трудности в согласовании физического времени с фиктивными временами, так как одному и тому же физическому времени соответствуют различные фиктивные времена для каждой планеты и возмущаемого тела.

Вычисление координат планет с помощью оскулирующих элементов устраняет вышеуказанную трудность, так как в этом случае нет необходимости в согласовании физического времени с фиктивными временами для координат больших планет.

Для решения системы дифференциальных уравнений (3) авторами разработан и реализован алгоритм с использованием метода Эверхарта [5]. Для определения области применимости данного алгоритма исследовалась эволюция орбит 10 короткопериодических комет.

Эволюция орбит вышеуказанных объектов на интервале времени 400 лет (18002200 гг.) исследовалась в работах [7]. Для численного интегрирования уравнений движения 10 короткопериодических комет использовался метод Эверхарта 27-го порядка с переменным шагом. При этом решение уравнений движения проводилось как путем совместного интегрирования, так и с использованием первого этапа регуляризации.

Сопоставление результатов вычислений, полученных путём совместного интегрирования уравнений движения больших планет и кометы, с результатами расчёта с учётом регуляризации и использованием оскулирующих элементов, не выявило существенных различий в элементах орбит, так как полученные расхождения находятся в пределах ошибок наблюдений.

Однако при интегрировании уравнений движения небесных тел, орбиты которых значительно отличаются от круговых, предпочтение следует отдавать методам с учётом регуляризации и использованием оскулирующих элементов больших планет, так как эти методы обладают лучшей устойчивостью и быстродействием.

Кроме того, следует отметить, что метод Эверхарта с учётом регуляризации и использованием банка данных оскулирующих элементов больших планет можно эффективно использовать для исследования эволюции орбит малых тел Солнечной системы, поскольку применение этого метода позволит значительно увеличить интервал интегрирования, а также сократит время проведенных исследований без существенной потери точности.

Работа выполнена в рамках Аналитической ведомственной челевой программой «Развитие научного потенциала высшей школь» (проект № РНП.2.1.1/745).

\section{БИБЛИОГРАФИЧЕСКИЙ СПИСОК}

1. Заусаев А.Ф., Заусаев Д.А. Численное интегрирование уравнений движения малых тел Солнечной системы с использованием оскулирующих элементов больших планет / В сб.: Тр. Шестой Всероссийской научн. конф. с междунар. участием. Ч. 3: Дифференциальные уравнения и краевые задачи / Мат. моделирование и краевые задачи. Самара: СамГТУ, 2009. - С. 125-130.

2. Standish E. M. JPL Planetary and Lunar Ephemerides DE405/LE405: Jet Prop Lab Technical Report. IOM 312. F-048, 1998.

3. Брумберг В. А. Релятивистская небесная механика. - М.: Наука, 1972. - 382 с.

4. Штифель E. Шейфеле Г. Линейная и регулярная небесная механика. - М.: Наука, 1975. - $304 \mathrm{c}$.

5. Everhart E. Implicit single methods for integrating orbits // Celestial Mechanics, 1974. T. $10 .-$ C. $35-55$.

6. Заусаев A. Ф., Заусаев A. А. Каталог орбитальной эволюции короткопериодических комет с 1800 по 2204 гг.. - М.: Машиностроение-1, 2007. - 410 с.

7. Заусаев А. Ф., Соловъев Л. А. Применение метода регуляризации к дифференциальным 
уравнениям движения комет // Вестн. Сам. гос. техн. ун-та. Сер. Физ.-мат. науки, 2009. 一 № 2(19). - С. 288-292.

MSC: 85-08

\section{NUMERICAL INTEGRATION OF CELESTIAL BODIES EQUATIONS WITH TAKING INTO ACCOUNT REGULARIZATION AND USE OSCULATING ELEMENTS OF MAJOR PLANETS}

\section{A. Zausaev, L. A. Solov'ev}

Samara State Technical University,

244, Molodogvardeyskaya st., Samara, 443100, Russia.

E-mail: zadmitriy@gmail.com

The regular differential equations for a problem of perturbed motion are received. The computing algorithm and the program of numerical integration of the equations of celestial motion by a method of Everhart taking into account regularization and use osculating of elements of major planets is developed.

Key words: numerical integration, regularisation, differential equation of motion, method of the osculation elements.

Original article submitted 30/VI/2010; revision submitted $03 / \mathrm{IX} / 2010$.

Dmitriy A. Zausaev, Postgraduate Student, Dept. of Applied Mathematics \& Computer Science. Leonid A. Solov'ev, Postgraduate Student, Dept. of Applied Mathematics \& Computer Science. 